with benzhexol. Risperidone is a newgeneration atypical antipsychotic agent with potent dopamine antagonist action (Owens, 1994). Despite this pharmacological effect, a low risk of extrapyramidal sideeffects has been reported. To our knowledge, this is the first report of rabbit syndrome caused by risperidone treatment.

Casey, D. E. (1992) Rabbit syndrome. In Movement Disorders in Neurology and Psychiotry (eds A. B. Joseph \& R. R. Young), pp. 139-142. Boston, MA: Blackwell Scientific Publications.

Owens, D. G. C. (1994) Extrapyramidal side effects and tolerability of risperidone: a review. journal of Clinical Psychiotry, 55 (suppl. 5), 29-35.

Schwartz, M., Weller, B., Endreich, M., et of (1995) Rabbit syndrome and tardive dyskinesia: two complications of chronic neuroleptic treatment. Journal of Clinical Psychiatry, 56. 212.

Todd, R., Lippman, S., Manshadi, M., et ol (1983) Recognition and treatment of rabbit syndrome, an uncommon complication of neuroleptic therapies. American Journal of Psychiatry, 140, 1519-1520.

M. Schwartz, A. Bemy, B. Sharf Bnai Zion Medical Center, Faculty of Medicine, 47 Golomb Street, PO Box 4940, Haifa 31048, Israel

\section{Jet lag and relapse of schizoaffective psychosis despite maintenance clozapine treatment}

Sir: Jet lag is a transient disorder of the sleep-wake schedule which results from rapid multiple time zone changes, affects only east-west travel and is worse after eastward travel. Its symptoms, which may last 7-10 days, include sleepiness, fatigue, exhaustion with insomnia during sleep period (Karacan et al, 1992).

There has been no report of de novo psychosis or relapse of a stabilised psychotic disorder following an acute jet lag. Tec (1981) reported a case of relapse of endogenous depression following jet lag. I present the case of a young man whose schizoaffective psychosis, stabilised on clozapine for five years, relapsed during an eastward transatlantic flight, despite continued compliance.

Mr A., a 26-year-old man, was first hospitalised in 1991 for a psychotic episode later diagnosed as schizoaffective disorder. Following failed trials of conventional neuroleptics he commenced clozapine on 3 December 1992. The Positive and Negative Syndrome Scale (PANSS; Kay et al, 1987) total score was 104. He was stabilised on clozapine $400 \mathrm{mg}$ per day and by early
1996 worked part-time in a grocery store. In December 1997, he left for Lebanon for a visit. Total PANSS score was 46, and maintenance dose of clozapine was $400 \mathrm{mg}$ h.s. After a six-hour flight to London, England, and before boarding the plane to Lebanon that day, he became guarded, suspicious and apprehensive. On arrival in Lebanon he became psychotic, with auditory hallucinations, ideas of reference and persecutory delusions that the police were after him. He saw a psychiatrist who increased clozapine to $500 \mathrm{mg}$ h.s., with benefit after two weeks.

His westward return trip to Canada a month later was uneventful. However, on arrival in Canada, the dose of clozapine had to be decreased to $450 \mathrm{mg}$ h.s. because of over-sedation.

This is the first case of psychotic relapse following acute jet lag. The dose of clozapine that controlled his psychosis in Lebanon (eastward travel) was excessive on return to Canada (westward travel). The clinical implication of this report is that such patients travelling eastward across time zones may need a slight dose increase to prevent a relapse.

Karacan, l., Williams, R. L. \& Moore, C. A. (1992) Sleep disorders: disorders of the sleep-wake schedule. In Comprehensive Textbook of Psychiotry, Vol. 2 (5th edn) (eds H. I. Kaplan \& B. J. Sadock), pp. III8-1119. Baltimore, MD: Williams \& Wilkins.

Kay, S. R., Fiszbein, A. Opler, L. A. (1987) The positive and negative syndrome scale (PANSS) for schizophrenia. Schizophrenia Bulletin, 13, 261-276.

Tec, L. (1981) Depression and jet lag. American Journal of Psychiatry, 138, 858.

\section{K. Oyewumi Clinical Evaluation Unit}

Program, London Psychiatric Hospital, PO Box 2532. Station A, 850 Highbury Avenue, London, Ontario, N6A 4HI, Canada

\section{Testicular pain and swelling on withdrawal of imipramine}

Sir: We present an unusual occurrence of testicular pain and swelling on withdrawal of imipramine. Testicular pain and swelling is noted in the package insert as an adverse reaction to the drug and it has been described in the literature with the use of desipramine (Deicken \& Carr, 1987; Thienhaus \& Vogel, 1988). A.B. is a 61year-old, married, unemployed male with major depression and generalised anxiety and had been treated with imipramine $150 \mathrm{mg}$ daily for the past eight years. He decided to stop imipramine because of prolonged adverse reactions. The medication was withdrawn over a two-week period. At the end of the first week he complained of bilateral pain, tenderness, swelling of testes and difficulty with micturition of gradual onset. The symptoms coincided with withdrawal.

Physical examination revealed bilateral swelling, redness and tenderness on palpation of testes. There was no history of being exposed to sexually transmitted diseases. On examination, his pulse and temperature was normal, there was no lymphadenopathy, no parotid swelling and no hypertrophy of prostate gland on rectal examination. Full blood count, erythrocyte sedimentation rate, urea, creatinine and electrolytes, prostate specific antigen, the tumour marker for beta human chorionic gonadotrophin, and alpha-fetoprotein, were all within normal limits. Urine microscopy was unremarkable and culture did not reveal any significant growth. Testicular ultrasound was not performed.

All of the genital symptoms and the other adverse symptoms subsided by the end of the fourth week and he remained symptom-free during regular follow-up over six months. The testicular pain and swelling may be due to an infection, but the laboratory findings did not support this hypothesis. There was a strong relationship between the time course of the withdrawal and the emergence of testicular pain and swelling. We postulate that a decline in level of the drug and/or its metabolite, possibly acting via an endocrine imbalance, gave rise to a hypersensitivity-type reaction and these symptoms subsided with restoration of equilibrium.

Deicken, R. F. \& Carr, R. E. (1987) Testicular pain and swelling with desipramine. Journal of Clinical Psychiatry, 48, 251-252.

Thienhaus, O. J. \& Vogel, N. (1988) Desipramine and testicular swelling in two patients. Journal of Clinical Psychiotry, 49, 33-34.

M. Perera, M. A. Khan Oldaker Street Clinic, PO Box I161, Devenport, TAS 7310, Australia

\section{Adults with Williams syndrome}

Sir: I read with interest the preliminary report on Williams syndrome (Davies et al, 1998). I feel that the omission of significant behavioural phenotypes and the ascertainment bias has marred an otherwise 